\title{
Nuestra experiencia a cinco años y medio en TVT
}

\author{
A. Hualde Alfaro, J. Jiménez Calvo, C. Sarmiento Gómez, M. Pinos Paul, A. de Pablo Cárdenas, \\ L. Ripa Saldias, A. Rivas Alonso, M. Ruiz Ramo, A. Santiago González de Garibay
}

Servicio de Urología. Hospital Virgen del Camino. Pamplona.

Actas Urol Esp. 2006; 30 (2): 181-185

\section{RESUMEN}

NUESTRA EXPERIENCIA A CINCO AÑOS Y MEDIO EN TVT

Objetivo: Presentar los resultados de eficacia de TVT a cinco años en términos de curación de incontinencia y complicaciones para el tratamiento de la IUE y de satisfacción personal con el resultado de la intervención.

Material y métodos: Un total de 369 incontinentes con una edad media de 59,9 años fueron implantadas con el sistema TVT en nuestro hospital pacientes desde 1998 hasta 2003. De ellas, 326 pacientes (85\%) presentaban IUE pura y 56 (15\%) incontinencia mixta con predominio de esfuerzo. La evaluación preoperatorio se realizó mediante historia clínica, exploración física, prueba de esfuerzo, flujometría y medición residuo postmiccional. La cistomanometría sólo se indicó en pacientes con urgencia o antecedentes de cirugía previa. El grado de severidad de la incontinencia se catalogó clínicamente. Como criterio de curación completa se consideró la ausencia de pérdidas objetivas y subjetivas. Se realizó encuesta telefónica para estudiar el grado de satisfacción de la paciente.

Resultados: El seguimiento medio fue de 35 meses con un rango entre 6 y 67 meses. Del total de pacientes, 317 presentaban continencia completa, lo que representa un $86 \%$. Por el contrario, 52 pacientes (14\%) presentaban escapes de orina. La tasa de urgencia de novo fue del 7\% y la de corte de TVT por obstrucción de 5,7\%. El porcentaje de reintervenciones, con sólo un caso de hematoma masivo.

Conclusiones: Hasta ahora se ha considerado siempre como patrón oro de las intervenciones de incontinencia de la orina, la técnica suprapúbica de Bursch, quizás la más contrastada en el tiempo, pero en la actualidad está siendo sustituida por las técnicas de malla suburetral sin tensión, cuya principal ventaja, es la simplicidad del método, su menor costo económico y la mayor comodidad para la paciente, pues convertimos una cirugía con varios días de ingreso, en una cirugía ambulatoria, con unos resultados hasta el momento similares en tasa de curación, Pero habrá que comprobar que los resultados a largo plazo de las mallas, si se mantienen en el tiempo y no aparecen complicaciones derivadas de la malla.

Palabras clave: TVT. Incontinencia urinaria de esfuerzo.

\section{ABSTRACT}

TVT OUR EXPERIENCE FIVE YEARS AN SIX MONTHS LATER

Objective: We present the outcome of urinary incontinence surgery after TVT more than five year of follow-up. Efficacy was evaluated in terms of complete cure of incontinence, complications, and patient satisfaction.

Material y methods: A sample of 369 female with genuine stress incontinence or mixed incontinence who had a TVT performed in our hospital between 1998-2003 were evaluated. Sample average age was 59.9 years. A total of 326 patients (85\%) had genuine stress incontinence and 56 (15\%) mixed incontinence. Preoperative evaluation was base on: clinical history and physical examination, stress test, flowmetry and post voided residual. Cistometry was indicated only in patients with previous surgery and mixed incontinence. Severity of incontinence was graded clinically. Primary outcome measure was complete cure of incontinence defined as lack of objective and subjective leakage. In addition a phone survey about patient satisfaction with the surgery was carried out.

Results: The average follow up was 35 months (6-67). A total of 317 were complete continent (86\%). By contrast, 52 patients (14\%) had leakages jet. The novo urgency rate was $7 \%$ and a $5,7 \%$ of patients were reoperated to cut the mesh because of obstruction. Nevertheless, the reoperation rate was low and only a patient showed a massive haematoma which needed to be evacuated.

Conclusión: Bursch Technique through suprapubic route has been classically considered the gold standard procedure for stress urinary incontinence for the last decades, having been substituted nowadays by tension-free suburethral mesh sling whose most important advantages are: Simplicity of the method, lower cost and higher comfort for the patient due to the fact that we have chaged in-patient surgery program for ambulatory one with similar results in order to sanation.

Anyway we will have to check if the preliminary good results and no complications persist in the follow-up.

Keywords: TVT. Stress urinary incontinence. 
$\mathrm{E}$ fundamento terapéutico del procedimiento tension free vaginal tape (TVT) para el tratamiento de la incontinencia urinaria de esfuerzo (IUE), se basa en la teoría integral de la continencia concebida por Peter Petros y Ulmsten ${ }^{1}$. Dicha teoría, se desarrolló con el objetivo de explicar de forma integral los mecanismos fisiopatológicos involucrados, no solamente en la IUE, sino de los síntomas habitualmente coexistentes, tales como la urgencia, polaquiuria, nicturia, alteraciones del vaciamiento vesical e intestinal, además del dolor pélvico crónico. Esa teoría considera que dichos sintomas son interdependientes, dentro de un proceso fisiopatológico común. Básicamente, la IUE, la urgencia y la disfunción de vaciado vesical se presentan por alteraciones en los elementos de soporte suburetral, los ligamentos y los músculos del suelo pélvico. Los defectos en la tensión aplicada por los músculos y ligamentos sobre las fascias yuxtapuestas a la pared vaginal determinan la apertura o el cierre del cuello vesical y de la uretra. Los mecanismos de cierre uretral en la mujer se localizan principalmente en su porción media. La falta de soporte por parte de los ligamentos pubouretrales en la uretra media y el deterioro de la función e inserción de los músculos pubococcígeos predisponen a las pacientes a presentar IUE.

Desde su descripción e introducción en la práctica clínica entre 1994 y 1995 en los países nórdicos, se han colocado un total de 150.000 unidades. En Europa, los países donde más se han implantado han sido en Francia (40.000) y Alemania (20.000).

Aunque en España su introducción y desarrollo se realizó masivamente a partir del año 2000, nosotros comenzamos a utilizarla a finales de 1998. Por lo que, el objetivo de este estudio es presentar los resultados de eficacia a cinco años en términos de curación de incontinencia y complicaciones en una serie amplia de pacientes intervenidas por IUE. Además, se presentan los resultados de una encuesta telefónica de satisfacción personal con el resultado de la intervención.

\section{MATERIAL Y MÉTODOS}

\section{Pacientes}

La muestra consta de 369 incontinentes con una edad media de 59,9 años intervenidas en nuestro hospital pacientes desde 1998 hasta 2003. De ellas, 326 pacientes (85\%) presentaban IUE pura y 56 (15\%) incontinencia mixta con predominio de esfuerzo.
Diagnóstico y evaluación funcional

Previa a la indicación quirúrgica, las pacientes fueron estudiadas mediante historia clínica completa y exploración física cuidadosa ginecológica para objetivar la existencia de cistocele, rectocele, colpocele. Además, se realizó una prueba de esfuerzo para objetivar las pérdidas, un test de Boney, una evaluación de la función de los músculos pelvianos y un q-tip test para evaluar la movilidad uretral. La valoración del cistocele y cuantificación del grado de cistocele fue especialmente cuidadosa dadas sus implicaciones quirúrgicas. Se consideró cistocele grado I cuando no alcanzaba el introito, grado II cuando este llega hasta introito vaginal y, grado III cuando pasa más allá del introito vaginal. La valoración del colpocele y la indicación de histerectomía vaginal fueron realizadas por el servicio de ginecología. Ya que, es el encargado de realizarla en el caso de estar indicada.

La evaluación funcional básica consistió en la realización de flujometría y valoración ecográfica del residuo postmiccional. La cistomanometría de llenado y vaciado no se realizó de forma sistematizada. Aquellas paciente que presentaban IUE pura no se les practicó excepto en casos esporádicos. Sin embargo, si se practicó a todas aquellas que presentaban incontinencia mixta y/o antecedentes de cirugía previa antiincontinencia.

\section{Grado de severidad incontinencia}

Se realizó siguiendo una clasificación arbitraria y subjetiva. Si bien útil y práctica en la clínica diaria y que nos da una idea bastante cercana de la realidad de la severidad de la incontinencia. Se consideró como pacientes con incontinencia de mínimos esfuerzos a aquellas con pérdidas al andar. Pacientes con incontinencia moderada a aquellas con perdidas al toser o reír. Pacientes con incontinencia a grandes esfuerzos cuando las pérdidas se presentaban al coger pesos importantes o al hacer ejercicio físico.

\section{Estudio del impacto de la incontinencia sobre calidad de vida}

Se realizó mediante una escala numérica del 0 al 10. Solicitamos a la paciente que nos señalase el impacto que su incontinencia urinaria le afectaba a su calidad de vida. Indicándole que el 0 correspondía a una situación en la que no le afectaba nada a su calidad de vida y 10 cuando estaba muy afectada y le restringía sus actividades normales. 


\section{Técnica quirúrgica}

El procedimiento quirúrgico ha sido el ya descrio ampliamente en la literatura científica sin variaciones. La cirugía fue llevada a cabo por todos los miembros del servicio. Aquellas pacientes que presentaban cistocele grado II o mayor se les realizaron corrección del mismo. Hasta enero del 2003, los cistoceles se trataron con una plastia anterior. Posteriormente a dicha fecha, se corrigieron con una malla de Prolene sin tensión tipo Ginemesh. A las pacientes con colpocele se les practicó histerectomía en el mismo acto quirúrgico por un ginecólogo. En estos casos, las pacientes fueron ingresadas a cargo del Servicio de Ginecología y quedaron a su cargo. Si bien, nosotros llevamos a cabo la cirugía antiincontinencia. En el periodo inicial de implantación del procedimiento, se indicó la colocación de TVT asociando a laparotomía para rechazar vejiga y controlar visualmente el paso de las agujas por el espacio retropúbico a 4 pacientes con antecedentes de cirugía retropúbica antiincontinencia. De esta forma, se evitó la posible perforación vesical. Posteriormente, una vez ganada experiencia, se realizó la colocación de TVT sin laparotomía a 13 pacientes más de la serie con el antecedente de cirugía previa retropúbica. En ninguno de estos casos se presentaron complicaciones ni intraoperatorias ni postoperatorias.

Desde al año 1999, las pacientes sin factores de riesgo y sin cirugía vaginal asociada, se intervinieron en régimen de cirugía mayor ambulatoria. En la actualidad, también se intervienen de forma ambulatoria a las pacientes con cistocele a las que se les coloca una malla de Ginemesh. Se les ingresa en el hospital por la mañana y se les da el alta a primera hora de la tarde con sonda. A las 24 horas se les citan a la consulta externa evaluar la herida, comprobar posibles sangrados y retirarles la sonda vesical. Antes de la retirada se llena la vejiga con suero fisiológico y se les invita a orinar para, posteriormente, realizar una evaluación ecográfica del residuo postmiccional. En caso de retención, se le enseña a la paciente, o a un familiar, a realizar sondajes intermitentes hasta obtener residuos inferiores a $75 \mathrm{cc}$.

\section{Seguimiento y criterios de curación}

El esquema de seguimiento realizado tras la cirugía consistió en una visita al mes, a los 6 meses y, a partir de entonces, cada año. En cada visita se recogió información clínica sobre la efi- cacia de la cirugía, se realizó una prueba de esfuerzo y una exploración física detallada para evidenciar posibles complicaciones postquirúrgicas. En cuanto a los criterios de curación, se consideró curación objetiva a la ausencia de pérdida de orina en la prueba de esfuerzo con la vejiga llena y curación subjetiva a la referida por la paciente como ausencia de perdidas. Finalmente, se considero curación completa a las pacientes con ausencia de pérdidas de orina tanto subjetiva como objetiva con el esfuerzo o asociada a urgencia.

Estudio del grado de satisfacción post-quirúrgica Dado que, en ocasiones la historia clínica, realizada por todos lo miembros del servicio de manera heterogénea, no refleja bien la realidad y sobre todo el grado de satisfacción de las pacientes, se llevo a cabo el envió por correo de un cuestionario de calidad de vida (potenciani) y una encuesta telefónicas personal. En esta última, se recogió información sobre la situación clínica de las pacientes respecto a la continencia y la presencia de urgencia miccional. Además, se les preguntó si volvería a intervenirse para como forma indirecta de recoger información sobre el grado de satisfacción con la técnica quirúrgica.

\section{RESULTADOS}

Técnicas quirúrgicas

Se realizaron un total de 369 intervenciones de las cuales, 219 consistieron en implantes de TVT como único procedimiento (59\%). Si bien, en el periodo inicial de la técnica, en cuatro pacientes con antecedente de cirugía retropúbica el implante de la TVT se realizó junto a laparotomía como medida de seguridad para prevenir lesiones vesicales con el paso de las agujas. En 150 casos (41\%) se asoció otras técnicas complementarias para la corrección de cistocele, rectocele o la realización de una histerectomía (Tabla 1).

Tabla 1

Técnicas quirúrgicas realizadas en las 369 pacientes

\begin{tabular}{lcc}
\hline Técnica quirúrgica & Número & (\%) \\
\hline TVT simple & 219 & $(59)$ \\
TVT + Plastia anterior vaginal & 81 & $(22)$ \\
TVT + Plastia anterior vaginal con malla & 5 & $(1)$ \\
TVT + Histerectomía mas plastias vaginales & 64 & $(17)$
\end{tabular}

*4 casos con laparotomía asociada 


\section{Resultados globales de continencia}

De las 369 pacientes, 66 (18\%) se evaluaron a los 5 años de evolución, 60 (16\%) a los 4 años, 79 (21\%) a los 3 años, 88 (23\%) a los 2 años y 76 (21\%) al año. El seguimiento medio fue de 35 meses con un rango entre 6 y 67 meses. Del total de pacientes, 317 presentaban continencia completa, lo que representa un $85 \%$. Por el contrario, 52 pacientes (14\%) presentaban escapes de orina. En 31 de estas últimas $(8,4)$, los escapes estaban relacionados con el esfuerzo y en $21(5,8)$ lo eran precedidos de urgencia.

\section{Desaparición urgencia y urgencia de novo}

En cuanto a los resultados en el grupo de pacientes con incontinencia mixta previa a la cirugía, en 29 de las 56 pacientes (52\%) persistía la urgencia miccional tras la implantación del TVT. Por otra parte, la aparición de urgencia de novo se produjo en 28 pacientes (7\%).

\section{Resultados de segundas cirugías}

Un total de 30 pacientes habían sido intervenciones previamente con técnicas antiincontinencia (8\%). Por lo que, la implantación de TVT representaba un intento de solucionar una situación previa de fracaso. De estas, 25 pacientes consiguieron la continencia completa, lo que representa un $83 \%$ de buenos resultados para este grupo de pacientes más complejas. De los casos restantes, en dos persistía una IUE moderada $(7 \%)$ y en tres incontinencia mixta (10\%).

\section{Retención postoperatoria}

El tiempo medio de catéter vesical fue de 1,25 días, incluyendo como día de ingreso el de su permanencia en la Unidad de Cirugía Mayor Ambulatoria sin pernoctación. En 57 pacientes (15\%) se obtuvieron residuos postmiccionales superiores $75 \mathrm{cc}$. Por lo que, o bien se mantuvo la sonda por un periodo mayor de tiempo o se instauró pauta de autosondaje intermitente. En 15 pacientes (4\%) la retención se prolongó más allá de cinco días, en 21 (6\%) más allá de los 10 y, en 17 (5\%), más de 20. Sólo en un pequeño grupo de 4 pacientes (1\%), la retención se prolongó por un periodo superior a los 2 meses. En 21 (5,7\% de la muestra global) de estas pacientes hubo que realizar una nueva intervención para sección la malla por la excesiva tensión que provocaba obstrucción y urgencia miccional.

\section{Complicaciones}

La complicación más frecuente fue la detección de un hematoma sintomático durante el seguimiento. En total lo presentaron 15 pacientes lo que representan un 4,6\% de la muestra. El resto de complicaciones viene reflejadas en la Tabla 2. Los porcentajes son realmente bajos, no incluimos la perforación vesical durante el procedimiento como una complicación pues no parece mas bien una incidencia quirúrgica, no obstante en solo un paciente con cirugía previa hubo perforación vesical. La extrusión de malla se produjo en sólo tres pacientes.

\section{Reintervenciones}

En la Tabla 3 se exponen el número y porcentaje de las reintervenciones que fueron necesarias a lo largo del seguimiento. Como ya se ha comentado la más frecuente fue la sección de la malla por obstrucción en 21 pacientes.

\section{Resultados encuesta telefónica}

En total se realizaron 261 encuestas telefónicas por un solo miembro del servicio a pacientes con al menos año y medio de seguimiento. El porcentaje de respuesta fue variable para cada periodo de tiempo y no se pudo obtener información completa de todas las pacientes. En la Tabla 4 se muestran los resultados sobre situación de continencia y grado de satisfacción con la interven-

Tabla 2

Complicaciones

\begin{tabular}{lcc}
\hline Dolor pélvico crónico & 1 & $0,3 \%$ \\
Extrusión de malla & 3 & $0,8 \%$ \\
Reintervenciones por sangrado & 1 & $0,3 \%$ \\
Lesión uretral intraoperatoria & 1 & $0,3 \%$ \\
Transfunsiones. & 2 & $0,8 \%$ \\
Hematomas clínicos, de mas de $5 \mathrm{cms}$ & 15 & $4,6 \%$ \\
\hline
\end{tabular}

Tabla 3

Reintervenciones

\begin{tabular}{lcc}
\hline Cortes de TVT & 21 & $5,7 \%$ \\
Retirada de malla intrauretral & 1 & $0,3 \%$ \\
Retirada de malla a las 6 horas & 1 & $0,3 \%$ \\
Cirugia por hematoma & 1 & $0,3 \%$ \\
Evacuación de hematoma infectado & 1 & $0,3 \%$ \\
\hline
\end{tabular}


Tabla 4

Resultados: (Encuestas contestadas)

\begin{tabular}{ccccc}
\hline Años & $\begin{array}{c}\text { No } \\
\text { pacientes }\end{array}$ & \multicolumn{1}{c}{ I.U.E. } & Urgencia & Insatisfechas \\
\hline 5 & 47 & $3 p-6,5 \%$ & $6 p-12,7 \%$ & $4 p-8,5 \%$ \\
4 & 39 & $4 p-10 \%$ & $4 p-10 \%$ & $6 p-15 \%$ \\
3 & 52 & $5 p-9,6 \%$ & $15 p-24,5 \%$ & $9 p-17,9 \%$ \\
2 & 53 & $2 p-3,7 \%$ & $9 p-16,9 \%$ & $4 p-7,5 \%$ \\
1 & 76 & $3 p-4 \%$ & $11 p-14,4 \%$ & $7 p-9,2 \%$ \\
\hline
\end{tabular}

ción en los diferentes periodos de seguimiento considerados. La tasa media de incontinencia de esfuerzo fue de $6,7 \%$, la de incontinencia mixta de $7,5 \%$ y la urgencia de $12,2 \%$. La tasa media de incontinencia global, como ya se ha expuesto, alcanzó el 14\%. En cuanto al grado de satisfacción expresado como la intención de volverse a intervenir fue elevado. Sólo el 10,7\% de las pacientes se mostraron insatisfechas con el resultado operatorio y no volverían a intervenirse.

\section{DISCUSIÓN}

Cuando realizamos TVT simple o asociado a corrección de cistocele ha habido una variación a lo largo de estos cinco años, al principio las pacientes eran ingresadas en el hospital y permanecían en él hasta conseguir micción, posteriormente seguían ingresando pero eran dadas de alta a las 24 horas de la intervención sin sonda o con sondajes intermitentes en el caso de ser retencionistas y estos últimos dos años realizamos la intervención en régimen de cirugía mayor ambulatoria de tal forma que las pacientes salen del hospital a la tarde sin sonda, o con sondajes intermitentes en el caso de que tengan residuo postmiccional.

En nuestras manos los resultados a largo plazo de la técnica de TVT, nos parecen buenos para la incontinencia de esfuerzo, con unas cifras por debajo del 10\%, y casi siempre con pérdidas muy inferiores a la incontinencia previa, mantenida a lo largo de los años, estas cifras coinciden con la mayoría de los trabajos publicados ${ }^{3,5,6}$.

Creemos que el principal problema de la intervención con malla de Prolene suburetral, es la aparición de la urgencia miccional, asociada generalmente a cierto grado de obstrucción, aunque no siempre se objetiva en la urodinamia.
En la literatura la obstrucción está descrita entre el 3 y el $25 \%^{2,3}$ de los procedimientos, en nuestro caso es en un 15\%, en general bien tolerada, con tratamiento con anticolinérgicos para la urgencia miccional asociada, pero en 21 pacientes hemos realizado corte de TVT $(5,7 \%)$, por afectación clara de la calidad de vida de las pacientes, lo que nos coloca por encima de la mayoría de los trabajos que van del $1,2 \%$ al $3 \%$ de los procedimientos ${ }^{3,4,6}$.

Nuestra experiencia ya va para 6 años con la TVT, y para nosotros al menos, es el patrón oro, a mejorar en el aspecto de la urgencia miccional.

En nuestra opinión, las nueva técnicas de malla suburetral, (TOT), tienen su posible mejora respecto a la TVT clásica, en una menor urgencia de novo y en la ausencia de casos de muerte por lesión vascular o intestinal, manteniendo los resultados en la continencia.

\section{REFERENCIAS}

1. Petros P. Ulmsten U. An integral theory of female urinary incontinente. Experimental and clinical considerations. Acta Obstet Gynecol Scand 1990:153:7-31.

2. Nilson I. Kuuva N. Falconer C. Rezapour M. Ulmsten U. Long-term results of the tension free vaginal tape (TVT) procedure for treatment stress urinary incontinente. Internation Urogynecology Journal 2001. Supplement 12(4);6568.

3. Maser AR, Muñoz Garrido F, Maroun B, Viana P, Mattos I, Hernández García JM, et al. Resultados del tratamiento quirúrgico de la incontinencia urinaria mediante TVT. Segundo Congreso Nacional sobre disfunciones del suelo pelviano. Libro de ponencias 115-124.

4. Meschia M, Pifarotti P. Tension free vaginal tape: Analisis of outcomes and complications in 404 stress incontinent women. Intenational Urogynecology Journal And Pelvic flor Dysfunctión 2001;12( suppl.2):S2-S27.

5. Rezapour M. Ulmsten U. Tension free vaginal tape; in women with mixed urinary incontinence. A long term follow up. International Urogynecology Journal and Pelvic floor dysfunction. 2001;12( Suppl 2):S15-18.

6. Gutiérrez Baños, JL, Martín García B, Portillo Martín JA, Del Valle Schaan JI, Hernández Rodríguez R, et al. Tratamiento de la incontinencia urinaria de esfuerzo mediante el implante suburetral de cintas libres de tension (TVT). 4 anos de experiencia. Arch-Esp-Urol. 2003 oct;56(8): 885-892.

Dr. A. Hualde Alfaro

Servicio de Urología. Hospital Virgen del Camino. Irunlarrea, 2 - 31008 Pamplona. Navarra E-mail: antonio_hualde@yahoo.es

(Trabajo recibido el 16 de enero 2006) 